\title{
The effects of a magnetic barrier and a nonmagnetic spacer in tunnel structures
}

\author{
Ali A. Shokri ${ }^{1 *}$ and Alireza Saffarzadeh ${ }^{2} \dagger$ \\ ${ }^{1}$ Department of Physics, Sharif University of Technology, 11365-9161, Tehran, Iran \\ 2 Department of Physics, Tehran Payame Noor University, Fallahpour St., Nejatollahi St., \\ Tehran , Iran
}

(November 21, 2018)

The spin-polarized transport is investigated in a new type of magnetic tunnel junction which consists of two ferromagnetic electrodes separated by a magnetic barrier and a nonmagnetic metallic spacer. Based on the transfer matrix method and the nearly-free-electron-approximation the dependence of the tunnel magnetoresistance (TMR) and electron-spin polarization on the nonmagnetic layer thickness and the applied bias voltage are studied theoretically. The TMR and spin polarization show an oscillatory behavior as a function of the spacer thickness and the bias voltage. The oscillations originate from the quantum well states in the spacer, while the existence of the magnetic barrier gives rise to a strong spin polarization and high values of the TMR. Our results may be useful for the development of spin electronic devices based on coherent transport.

\footnotetext{
*E-mail: aashokri@mehr.sharif.edu

†Corresponding author. E-mail: a-saffar@tehran.pnu.ac.ir
} 


\section{INTRODUCTION}

During recent years, the large magnetoresistance of magnetic tunnel junctions (MTJs) has attracted much attention due to the possibility of its application in digital storage and magnetic sensor technologies [1,2]. In most of the experiments, the MTJs consist of two ferromagnetic metal (FM) electrodes separated by a thin insulator (FM/I/FM). In these structures, the electrical resistance is reduced when the magnetization direction of the FM layers changes from antiparallel to parallel alignment by an external magnetic field. Because the tunnel magnetoresistance (TMR) strongly depends on the spin polarization of the magnetic electrodes $[3,4]$, attempts have been made to fabricate junctions with more highly spin-polarized ferromagnets $[5,6]$.

Recent experiments on the TMR have shown that the insertion of a thin nonmagnetic metal (NM) layer between insulating barrier and the FM electrode (FM/I/NM/FM) reduces the TMR, when thickness of the NM layer increases [7-10]. This decrease of the TMR can be attributed to the decoherence tunneling of electrons from one electrode to the other through the NM layer [11]. On the other hand, recent observations by Yuasa et al [12] show clear oscillations of the TMR in high-quality $\mathrm{NiFe} / \mathrm{Al}_{2} \mathrm{O}_{3} / \mathrm{Cu} / \mathrm{Co}$ junctions in which the $\mathrm{Cu} / \mathrm{Co}$ is a single crystal. This oscillatory behavior has been interpreted in terms of the formation of spin-polarized resonant tunneling.

The effect of nonmagnetic metallic interlayers has been theoretically investigated in FM/NM/I/NM/FM structures. Based on the Kubo formalism, Vedyayev et al. [13] studied magnetoconductivity as a function of NM layer thickness at low bias voltages. By using the transfer matrix approach, Wilczynski and Barnas [14] calculated the thickness and voltage dependence of TMR. These calculations showed that the presence of thin NM spacers can lead to the formation of quantum well states that lead to oscillations of magnetoconductivity and TMR. When the thickness of only one of the NM layer varies, and also in the case of an asymmetric structure, such as FM/NM/I/FM [15], the sign of magnetoconductivity and TMR is predicted to oscillate as a function of the NM layer thickness. 
TMR has also been investigated both experimentally [16,17] and theoretically [18-21] in spin filter tunnel junctions in which a ferromagnetic semiconductor (FMS) is applied as a

magnetic barrier. The large spin polarization achievable using magnetic barriers [22] makes spin filtering a nearly ideal method for spin injection into semiconductors, enabling novel spintronic devices [23]. When an FMS layer which acts as a spin filter is used in tunnel structures, due to the spin splitting of its conduction band, tunneling electrons see a spindependent barrier height. Thus one spin channel will have a larger tunneling probability than the other, and a highly spin-polarized current may result.

The purpose of the present work is to investigate theoretically the effect of a magnetic barrier on spin transport in the presence of a nonmagnetic spacer layer. Using the transfer

matrix method and the nearly-free-electron approximation we will study the variation of TMR and spin polarization of tunneling electrons in terms of the spacer thickness and bias voltage at $T=0 \mathrm{~K}$. We assume that the electron wave vector parallel to the interfaces and the electron spin are conserved in the tunneling process through the whole system.

The paper is organized as follows. In section 2, the model and the analytic formulae of the spin polarization and TMR for FM/FMS/NM/FM structure are presented. In section 3, numerical results obtained for a typical tunnel junction are discussed. The results of this work are summarized in section 4.

\section{DESCRIPTION OF MODEL AND FORMALISM}

We consider two semi-infinite FM electrodes separated by a ferromagnetic barrier which acts as a spin filter, and a nonmagnetic metallic layer as a quantum well, in the presence of DC applied bias $V_{a}$ as shown in Fig. 1. For simplicity, we assume that the two FM electrodes are made of the same metal and all of the interfaces are flat. In a nearly-freeelectron approximation of spin-polarized conduction electrons, the longitudinal part of the effective one-electron Hamiltonian can be written as 


$$
H_{x}=-\frac{\hbar^{2}}{2 m_{j}^{*}} \frac{d^{2}}{d x^{2}}+U_{j}(x)-\mathbf{h}_{j} \cdot \sigma+V^{\sigma},
$$

where $m_{j}^{*}(j=1-4)$ is the electron effective mass in the $j$ th layer, and

$$
U_{j}(x)=\left\{\begin{array}{cc}
0, & x<0 \\
E_{\mathrm{FL}}+\phi-e V_{a} x / t_{\mathrm{bar}}, & 0<x<t_{\mathrm{bar}} \\
-e V_{a}, & t_{\mathrm{bar}}<x<t_{\mathrm{bar}}+t_{\mathrm{NM}}, \\
-e V_{a}, & x>t_{\mathrm{bar}}+t_{\mathrm{NM}},
\end{array}\right.
$$

where $E_{\mathrm{FL}}$ is the Fermi energy in the left electrode. $-\mathbf{h}_{j} \cdot \sigma$ is the internal exchange energy where $\mathbf{h}_{j}$ is the molecular field in the $j$ th FM electrode and $\sigma$ is the conventional Pauli spin operator. The last term in Eq. (1) is a spin-dependent potential and denotes the $s-f$ exchange coupling between the spin of tunneling electrons and the localized $f$ spins in the magnetic barrier. This term, within the mean-field approximation, is proportional to the thermal average of the $f$ spins, $\left\langle S_{z}\right\rangle$, and can be written as $V^{\sigma}=-\sigma I\left\langle S_{z}\right\rangle$. Here, $\sigma= \pm 1$ which corresponds to $\sigma=\uparrow, \downarrow$, and $I$ is the $s-f$ exchange constant in the magnetic barrier.

The Schrödinger equation for a biased barrier layer can be simplified by a coordinate transformation whose solution is the linear combination of the Airy function $\operatorname{Ai}[\rho(x)]$ and its complement $\operatorname{Bi}[\rho(x)][24]$. Considering all four regions of the FM/FMS/NM/FM junction shown in Fig. 1, the eigenfunctions of the Hamiltonian (1) with eigenvalue $E_{x}$ have the following forms:

$$
\psi_{j, \sigma}(x)=\left\{\begin{array}{cc}
A_{1 \sigma} e^{i k_{1 \sigma} x}+B_{1 \sigma} e^{-i k_{1 \sigma} x}, & x<0, \\
A_{2 \sigma} \operatorname{Ai}\left[\rho_{\sigma}(x)\right]+B_{2 \sigma} \operatorname{Bi}\left[\rho_{\sigma}(x)\right], & 0<x<t_{\mathrm{bar}}, \\
A_{3 \sigma} e^{i k_{3} x}+B_{3 \sigma} e^{-i k_{3} x}, & t_{\mathrm{bar}}<x<t_{\mathrm{bar}}+t_{\mathrm{NM}}, \\
A_{4 \sigma} e^{i k_{4 \sigma} x}+B_{4 \sigma} e^{-i k_{4 \sigma} x}, & x>t_{\mathrm{bar}}+t_{\mathrm{NM}},
\end{array}\right.
$$

where,

$$
\begin{gathered}
k_{1 \sigma}=\sqrt{2 m_{1}^{*}\left(E_{x}+h_{0} \sigma\right)} / \hbar \\
k_{3}=\sqrt{2 m_{3}^{*}\left(E_{x}+e V_{a}+E_{\mathrm{F}, \mathrm{NM}}-E_{\mathrm{FL}}\right)} / \hbar,
\end{gathered}
$$




$$
k_{4 \sigma}=\sqrt{2 m_{4}^{*}\left(E_{x}+e V_{a}+\eta h_{0} \sigma\right)} / \hbar
$$

are the electron wave vectors along the $x$ axis. Here, $h_{0}=\left|\mathbf{h}_{j}\right|$ and $\eta=+1(-1)$ for parallel (antiparallel) alignment of the magnetizations. The coefficients $A_{j \sigma}$ and $B_{j \sigma}$ are constants to be determined from the boundary conditions, while

$$
\rho_{\sigma}(x)=\frac{x}{\lambda}+\beta_{\sigma},
$$

with

$$
\begin{gathered}
\lambda=\left[-\frac{\hbar^{2} t_{\mathrm{bar}}}{2 m_{2}^{*} e V_{a}}\right]^{1 / 3}, \\
\beta_{\sigma}=\frac{\left[E_{x}-E_{\mathrm{FL}}-\phi_{\mathrm{bar}}-V^{\sigma}\right] t_{\mathrm{bar}}}{e V_{a} \lambda} .
\end{gathered}
$$

Although the transverse momentum $\mathbf{k}_{\|}$is omitted from the above notations, the summation over $\mathbf{k}_{\|}$is carried out in our calculations.

Upon applying the boundary conditions such that the wave functions and their first derivatives are matched at each interface point $x_{j}$, i.e. $\psi_{j, \sigma}\left(x_{j}\right)=\psi_{j+1, \sigma}\left(x_{j}\right)$ and $\left(m_{j}^{*}\right)^{-1}\left[d \psi_{j, \sigma}\left(x_{j}\right) / d x\right]=\left(m_{j+1}^{*}\right)^{-1}\left[d \psi_{j+1, \sigma}\left(x_{j}\right) / d x\right]$, we obtain a matrix formula that connects the coefficients $A_{1 \sigma}$ and $B_{1 \sigma}$ with the coefficients $A_{4 \sigma}$ and $B_{4 \sigma}$ as follows:

$$
\left[\begin{array}{c}
A_{1 \sigma} \\
B_{1 \sigma}
\end{array}\right]=M_{\text {total }}\left[\begin{array}{c}
A_{4 \sigma} \\
B_{4 \sigma}
\end{array}\right],
$$

where

$$
\begin{aligned}
M_{\text {total }}= & \frac{k_{4 \sigma}}{k_{1 \sigma}}\left[\begin{array}{cc}
i k_{1 \sigma} & \frac{1}{\lambda} \frac{m_{1}^{*}}{m_{2}^{*}} \\
i k_{1 \sigma} & -\frac{1}{\lambda} \frac{m_{1}^{*}}{m_{2}^{*}}
\end{array}\right]\left[\begin{array}{cc}
\operatorname{Ai}\left[\rho_{\sigma}(x=0)\right] & \operatorname{Bi}\left[\rho_{\sigma}(x=0)\right] \\
\operatorname{Ai}^{\prime}\left[\rho_{\sigma}(x=0)\right] & \operatorname{Bi}^{\prime}\left[\rho_{\sigma}(x=0)\right]
\end{array}\right] \\
& \times\left[\begin{array}{cc}
\operatorname{Ai}\left[\rho_{\sigma}\left(x=t_{\mathrm{bar}}\right)\right] & \operatorname{Bi}\left[\rho_{\sigma}\left(x=t_{\mathrm{bar}}\right)\right] \\
\frac{1}{\lambda} \frac{1}{m_{2}^{*}} \operatorname{Ai}^{\prime}\left[\rho_{\sigma}\left(x=t_{\mathrm{bar}}\right)\right] & \frac{1}{\lambda} \frac{1}{m_{2}^{*}} \operatorname{Bi}^{\prime}\left[\rho_{\sigma}\left(x=t_{\mathrm{bar}}\right)\right]
\end{array}\right] \\
& \times\left[\begin{array}{cc}
\cos \left(k_{3} t_{\mathrm{NM}}\right) & -\frac{m_{3}^{*}}{k_{3}} \sin \left(k_{3} t_{\mathrm{NM}}\right) \\
\frac{k_{3}}{m_{3}^{*}} \sin \left(k_{3} t_{\mathrm{NM}}\right) & \cos \left(k_{3} t_{\mathrm{NM}}\right)
\end{array}\right]\left[\begin{array}{cc}
i k_{4 \sigma} & m_{4}^{*} \\
i k_{4 \sigma} & -m_{4}^{*}
\end{array}\right]^{-1} \\
& \times\left[\begin{array}{cc}
e^{-i k_{4 \sigma}\left(t_{\mathrm{bar}}+t_{\mathrm{NM}}\right)} & 0 \\
0 & e^{i k_{4 \sigma}\left(t_{\mathrm{bar}}+t_{\mathrm{NM}}\right)}
\end{array}\right]^{-1}
\end{aligned}
$$


Since there is no reflection in region 4 , the coefficient $B_{4 \sigma}$ in Eq. (3) is zero and the transmission coefficient (TC) of the spin $\sigma$ electron, which is defined as the ratio of the transmitted flux to the incident flux can be written as

$$
T_{\sigma}\left(E_{x}, V_{a}\right)=\frac{k_{4 \sigma} m_{1}^{*}}{k_{1 \sigma} m_{4}^{*}}\left|\frac{1}{M_{\text {total }}^{11}}\right|^{2},
$$

where $M_{\text {total }}^{11}$ is the left-upper element of the matrix $M_{\text {total }}$ defined in Eq. (11). At $T=0 \mathrm{~K}$, the spin-dependent current density for the magnetic tunnel junction at a given applied bias $V_{a}$ within the nearly-free-electron model is given by the formula [25]:

$$
J_{\sigma}\left(V_{a}\right)=\frac{e m_{1}^{*}}{4 \pi^{2} \hbar^{3}}\left[e V_{a} \int_{E_{0}^{\sigma}}^{E_{\mathrm{FL}}-e V_{a}} T_{\sigma}\left(E_{x}, V_{a}\right) d E_{x}+\int_{E_{\mathrm{FL}}-e V_{a}}^{E_{\mathrm{FL}}}\left(E_{\mathrm{FL}}-E_{x}\right) T_{\sigma}\left(E_{x}, V_{a}\right) d E_{x}\right],
$$

where $E_{0}^{\sigma}$ is the lowest possible energy that will allow transmission and is given by $E_{0}^{\uparrow}=$ $\max \left\{-h_{0},-e V_{a}-\eta h_{0}\right\}$ for spin-up and $E_{0}^{\downarrow}=h_{0}$ for spin-down electrons. It is clear that the tunnel current is modulated by the magnetic configurations of the both FM electrodes.

The degree of spin polarization for the tunnel current is defined by

$$
P=\frac{J_{\uparrow}-J_{\downarrow}}{J_{\uparrow}+J_{\downarrow}}
$$

where $J_{\uparrow}\left(J_{\downarrow}\right)$ is the spin-up (spin-down) current density. For the present structure, we obtain this quantity when the magnetizations of two FM electrodes and the FMS layer (magnetic barrier) are in parallel alignment. For calculating the TMR, both spin currents

in the parallel and antiparallel alignments are calculated. In this case, the TMR can be defined as

$$
\mathrm{TMR}=\frac{\left(J_{\uparrow}^{\mathrm{P}}+J_{\downarrow}^{\mathrm{P}}\right)-\left(J_{\uparrow}^{\mathrm{AP}}+J_{\downarrow}^{\mathrm{AP}}\right)}{J_{\uparrow}^{\mathrm{AP}}+J_{\downarrow}^{\mathrm{AP}}},
$$

where $J_{\uparrow, \downarrow}^{\mathrm{P}(\mathrm{AP})}$ corresponds to the current density in the parallel (antiparallel) alignments of the magnetizations in the FM electrodes, for a spin-up $(\uparrow)$ or spin-down $(\downarrow)$ electron.

\section{NUMERICAL RESULTS AND DISCUSSION}

Numerical calculations have been carried out to investigate the effect of a magnetic barrier and an NM metallic layer on the spin transport in the Fe/EuS/Au/Fe structure as 
a typical MTJ. We have chosen Fe and EuS because they have cubic structures and the lattice mismatch is very small [26]. The parameters $E_{\mathrm{F}}$ and $h_{0}$ for Fe electrodes are taken corresponding to $k_{\mathrm{F} \uparrow}=1.09 \AA^{-1}$ and $k_{\mathrm{F} \downarrow}=0.42 \AA^{-1}$ (for itinerant $d$ electrons) [27]. For the EuS layer we use $S=7 / 2, I=0.1 \mathrm{eV}[28], \phi=1.94 \mathrm{eV}[21]$ as a symmetric barrier height, and $t_{\mathrm{bar}}=1.3788 \mathrm{~nm}$ which corresponds to four monolayers of EuS $\langle 111\rangle$ [29]. The Fermi energy in the $\mathrm{Au}$ layer is $E_{\mathrm{F}, \mathrm{NM}}=5.51 \mathrm{eV}$ [30]. In practice, the effective masses of electrons may differ from that of free electron, but here for simplicity, we assume all electrons have the same mass, $m_{e}$ as free electrons.

In our considered system, the magnetization direction of the left Fe electrode and the EuS magnetic barrier stays fixed, because they are coupled through the interface. Inserting the NM spacer layer between the FMS layer and the right FM electrode decreases the exchange coupling between the two FM electrodes. Therefore the right Fe electrode is free and may be switched back and forth by an external magnetic field (see Fig. 1). In the following, we show the numerical results at $T=0 \mathrm{~K}$, so in the magnetic barrier we have $\left\langle S_{z}\right\rangle=S$.

The TC for tunneling electrons at the Fermi energy, and the TMR in the tunnel junction as a function of the NM layer thickness are shown in Figs. 2(a) and 2, respectively. It can be seen that both the TC and the TMR oscillate with the NM layer thickness due to the dependence of these quantities on the incident electron's energy and the NM layer thickness. The physics behind this behavior is easy to understand. When the energy of the incident electrons is approximately equal to the energy of a quasibound state in the quantum well, a resonance condition is fulfilled and the TC of the electrons through the structure strongly increases. On the other hand, the position of the quantum well levels, formed in the NM layer, strongly depends on the well thickness. Therefore, with continuous variation of the $t_{\mathrm{NM}}$, the position of the resonant states varies and this leads to the oscillations of the TC and the TMR. From Fig. 2, it is clear that where the transmission is high, the TMR is low. From the continuous variation of $t_{\mathrm{NM}}$, one can see that both the TC and the TMR oscillate with a period of $0.26 \mathrm{~nm}$ which is in excellent agreement with $\lambda_{F} / 2$, where $\lambda_{F}$ is the Fermi wavelength in the Au layer and is equal to $0.522 \mathrm{~nm}$. The results show that, for the spin-up 
electrons, the TC is very higher than the spin-down ones both in the parallel and antiparallel alignment of magnetizations. The reason can be explained by the spin filtering effect of the FMS barrier. As shown in Fig. 1, due to the spin splitting of the conduction band in the FMS layer, the barrier height for spin-up electrons is lowered and the tunneling probability greatly increases, while for the spin-down electrons the barrier height is raised and hence, the tunneling probability for these electrons reduces. Thus, a large spin-polarized current is produced.

In Fig. 2(b) we have also shown the TMR in terms of thickness, when $t_{\mathrm{NM}}$ changes in monolayer steps. At the monolayer thickness $t_{\mathrm{ML}, \mathrm{NM}}=0.2355 \mathrm{~nm}$, which corresponds to interlayer distance of $\mathrm{Au}\langle 111\rangle$, the TMR first increases in the first three monolayer and after that the TMR becomes less and starts to oscillate with a period of approximately $2.5 \mathrm{~nm}$. In contrast, for $t_{\mathrm{ML}, \mathrm{NM}}=0.27 \mathrm{~nm}$, the TMR initially decreases and the period of oscillations is approximately $7 \mathrm{~nm}$.

The voltage dependence of the TMR for several thicknesses is shown in Fig. 3, when $t_{\mathrm{ML}, \mathrm{NM}}=0.2355 \mathrm{~nm}$. We can see that, due to the FMS layer, the TMR is asymmetric, as a tunnel junction without a nonmagnetic layer is used. For each fixed voltage, the TMR oscillates, as is shown in Fig. 2(b) for $V_{a}=50 \mathrm{mV}$. The results show that more than $250 \%$ TMR can be obtained in reverse bias. By increasing the number of nonmagnetic monolayers, the TMR shows an oscillatory behavior in the positive voltages. Under a forward bias, electrons at the Fermi energy of the left electrode, tunnel into the empty states of the quantum well, which have been created due to the applied bias. Therefore the applied bias has a strong influence on the TMR and the oscillatory behavior will appear. On the other hand, under a reverse bias, electrons at the Fermi energy of the quantum well tunnel into the empty states of the left electrode. In this case, variations of the applied bias cannot strongly affect the TMR.

In order to further see the effect of the FMS layer on the tunnel currents, we have shown in Fig. 4 the spin polarization of tunneling electrons as a function of the bias voltage, for $t_{\mathrm{ML}, \mathrm{NM}}=0.2355 \mathrm{~nm}$. It is obvious that the spin polarization is symmetric for $t_{\mathrm{ML}, \mathrm{NM}}=0$, and 
becomes strongly asymmetric when the number of monolayers increases. The results show that the spin polarization can reach $99 \%$, which is evidence of spin filtering effect in the FMS layer.

The experimental measurements show that, if one uses a nonmagnetic insulator instead of a magnetic tunnel barrier, very low values for electron-spin polarization and TMR will result $[7,12]$. The oscillatory behavior of TMR in terms of the NM layer thickness, is qualitatively in agreement with the experiments of Yuasa et al [12], and shows that the coherent tunneling of electrons through the quantum well states is the main reason of this behavior.

The present results can be qualitatively compared with those already known in the relevant literature, when a nonmagnetic barrier is used. In $[13,14]$, the authors showed that in the $\mathrm{FM} / \mathrm{NM} / \mathrm{I} / \mathrm{NM} / \mathrm{FM}$ systems, the TMR in two different cases has an oscillatory behavior with NM layers: the case where the thickness of both NM layers are equally varied (symmetric case), and the case where only one of the NM layers is increased (asymmetric case). In the symmetric case, the TMR is always positive, but in the asymmetric case the TMR oscillates from positive to negative as a function of the NM layer thickness. The situation of the asymmetric case is similar to our structure in which only one NM layer has been used [15]. Thus, as it is clear from Figs. 2(b) and 3, our results are in qualitative agreement with the asymmetric case in FM/NM/I/NM/FM structures.

Finally, we discuss the effect of interface scattering on spin-injection from the FM into NM spacer [31,32]. For this purpose, we treated the magnetic barrier as a $\delta$-function $U_{\sigma} \delta(x)$, with $U_{\sigma}$ describing the barrier strength parameter for spin $\sigma$ electron. In this case, the parameter $U_{\sigma}$ is proportional to both height and width of the barrier. The numerical results showed that with increasing the $U_{\sigma}$, the TC reduces, and hence the spin-dependent currents decay exponentially, which is due to the interface resistance. On the other hand, the spin polarization and TMR increase with $U_{\sigma}$, and trend gradually towards constant values, because in these values of the barrier parameter, only the FM electrodes have a dominant influence on the spin polarization and TMR. 


\section{CONCLUDING REMARKS}

The magnetotransport behavior in an FM/FMS/NM/FM tunnel junctions was investigated theoretically, using the transfer matrix method and the nearly-free-electron approximation. The numerical results indicate that in $\mathrm{Fe} / \mathrm{EuS} / \mathrm{Au} / \mathrm{Fe}$ structure, the TMR has an oscillatory behavior as a function of the NM layer thickness. By increasing the thickness of the NM layer, both the TMR and electron-spin polarization, oscillate under the forward biases. Due to the presence of a magnetic barrier, there exist more than $250 \%$ TMR and 98\% spin polarization in the tunnel currents; this can be obtained by adjusting the thickness and the applied bias.

Our study restricted to the zero temperature limit, because the ferromagnetic transition temperature in most of the FMS layers is much lower than room temperature [29]. Low temperature spin transport is, however, important as a testing ground for novel ideas and concepts in future spin electronic devices based on coherent transport $[12,33]$.

\section{ACKNOWLEDGMENTS}

One of us (A.A.S.) would like to express his sincere thanks to Prof. K. Esfarjani for illuminative comments.

[1] Moodera J S, Kinder L R, Wong T M and Meservey R 1995 Phys. Rev. Lett. 743273

[2] Daughton J 1997 J. Appl. Phys. 813758

[3] Julliere M 1975 Phys. Lett. A 54225

[4] Maekawa S and Gäfvert U 1982 IEEE Trans. Magn. 18707

[5] Lu Y, Li X W, Gong G Q, Xiao G, Gupta A, Lecoeur P, Sun J Z, Wang Y Y and Dravid V P 1996 Phys. Rev. B 54 R8357 
[6] Seneor P, Fert A, Maurice J L, Montaigne F, Petroff F and Vaurès A 1999 Appl. Phys. Lett. 744017

[7] Moodera J S, Nowak J, Kinder L R, Tedrow P M, van de Veerdonk R J M, Smith B A, van Kampen M, Swagten H J M and de Jonge W J M 1999 Phys. Rev. Lett. 833029

[8] Sun J J and Freitas P P 1999 J. Appl. Phys. 855264

[9] LeClair P, Swagten H J M, Kohlhepp J T, van de Veerdonk R J M and de Jonge W J M 2000 Phys. Rev. Lett. 842933

[10] LeClair P, Kohlhepp J T, Swagten H J M and de Jonge W J M 2001 Phys. Rev. Lett. 861066

[11] Zhang S and Levy P M 1998 Phys. Rev. Lett. 815660

[12] Yuasa S, Nagahama T and Suzuki Y 2002 Science 297234

[13] Vedyayev A, Ryzhanova N, Lacroix C, Giacomoni L and Dieny B 1997 Europhys. Lett. 39219

[14] Wilczyński M and Barnaś J 2000 J. Appl. Phys. 885230

[15] Zhang W S, Li B Z and Zhang X 1998 J. Appl. Phys. 835332

[16] LeClair P, Ha J K, Swagten H J M, Kohlhepp J T, van de Vin C H and de Jonge W J M 2002 Appl. Phys. Lett. 80625

[17] Filip A T, LeClair P, Smits C J P, Kohlhepp J T, Swagten H J M, Koopmans B and de Jonge W J M 2002 Appl. Phys. Lett. 811815

[18] Worledge D C and Geballe T H 2000 J. Appl. Phys. 885277

[19] Wilczyński M, Barnaś J and Świrkowicz R 2003 J. Magn. Magn. Mater. 267391

[20] Saffarzadeh A 2003 J. Phys.: Condense Matter 153041

[21] Saffarzadeh A 2004 J. Magn. Magn. Mater. 269327

[22] Moodera J S, Hao X, Gibson G A and Meservey R 1988 Phys. Rev. Lett. 61637 
Hao X, Moodera J S and Meservey R 1990 Phys. Rev. B 428235

[23] Fiederling R, Keim M, Reuscher G, Ossau W, Schmidt G, Waag A and Molenkamp L 1999 Nature (London) 402787

[24] Abramowitz M and Stegun I A 1965 Handbook of Mathematical Functions (New York: Dover)

[25] Duke C B 1969 Tunneling Phenomena in Solids ed E Burstein and S Lundquist (New York: Plenum)

[26] Rücker U, Demokritov S, Arons R.R and Grünberg P 1996 J. Magn. Magn. Mater. 269-270 156

[27] Stearns M B 1977 J. Magn. Magn. Mater. 5167

[28] Nolting W, Dubil U and Matlak M 1985 J. Phys. C 183687

[29] Mauger A and Godart C 1986 Phys. Rep. 14151

[30] Kittel C 1986 Introduction to Solid State Physics (New York: John Wiley \& Sons) 6th ed.

[31] Blonder G E, Tinkham M and Klapwijk T M 1982 Phys. Rev. B 254515

[32] Hu C M and Matsuyama T 2001 Phys. Rev. Lett. 8766803

[33] Dietl T, Ohno H, Matsukura F, Cibert J and Ferrand D 2000 Science 2871019 


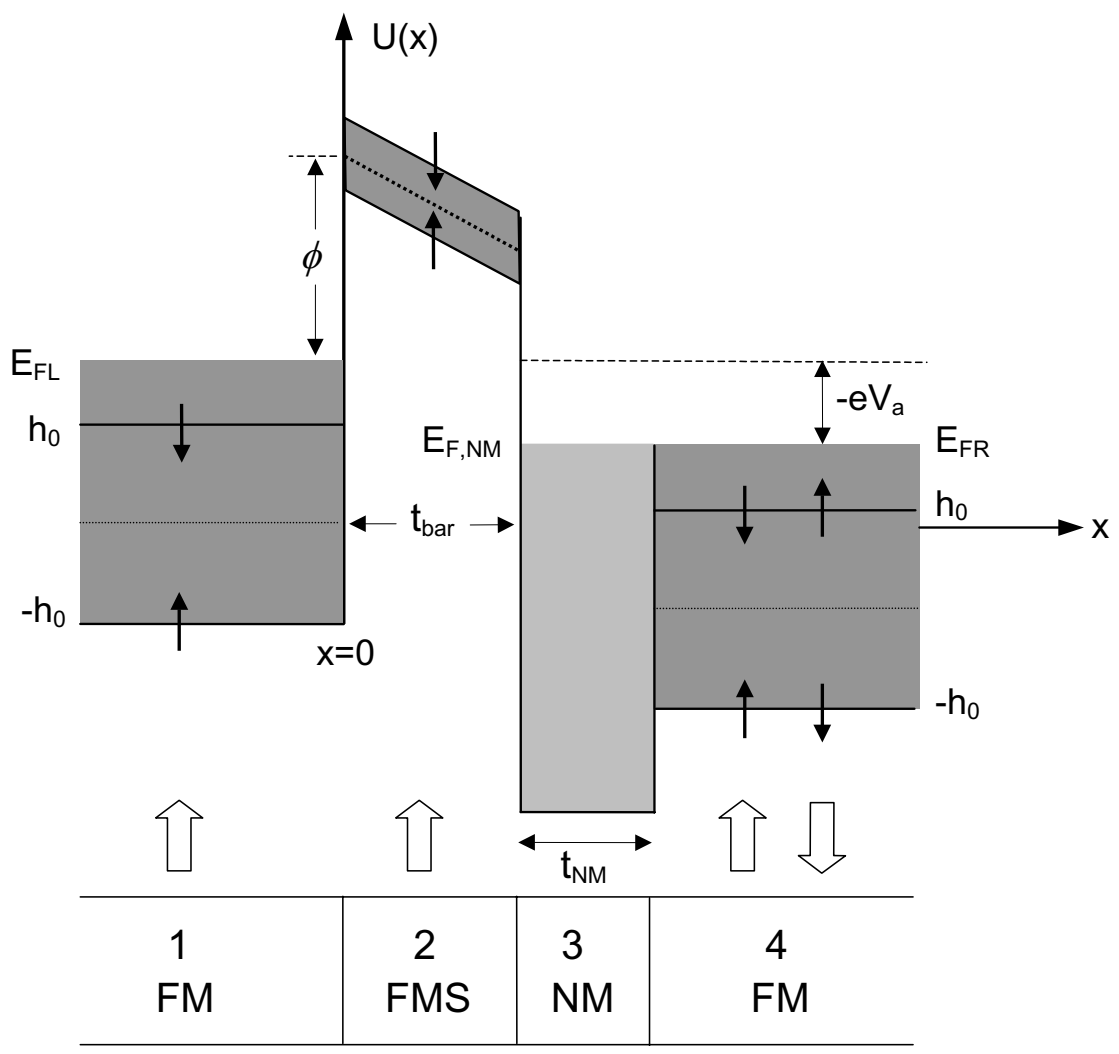

FIG. 1. Spin-dependent potential profile for an FM/FMS/NM/FM magnetic tunnel junction under forward bias $V_{a}$. In the FMS layer, the dashed line represents the bottom of the conduction band at $T \geq T_{C}$ and the thin arrows indicate the bottom of the conduction band for spin-up and spin-down electrons at $T<T_{C}$. The zero of energy is taken at the middle of the bottoms for the majority-spin band and minority-spin one in the left FM electrode. 

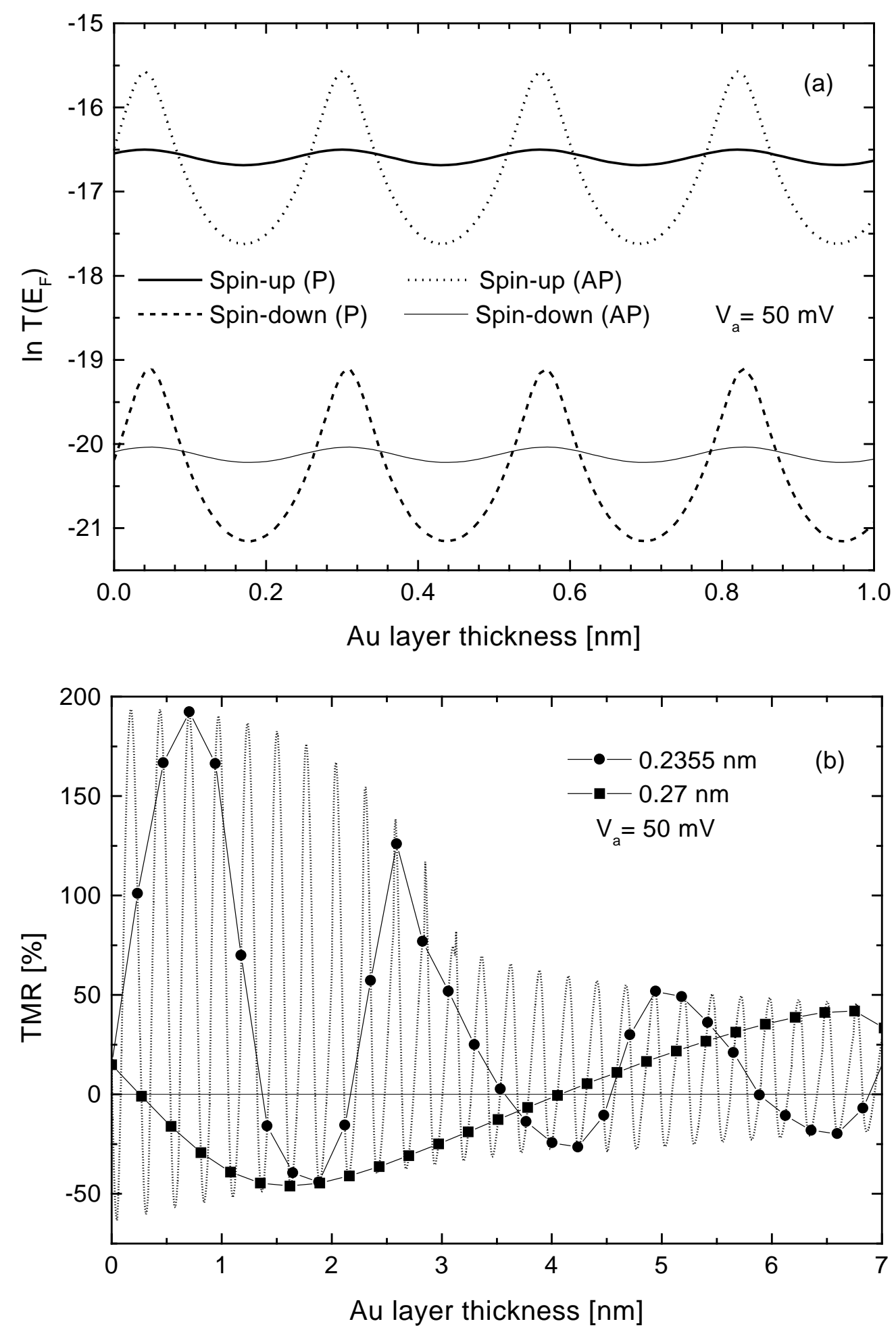

FIG. 2. (a) Spin-dependent TC at Fermi energy in the parallel (P) and antiparallel (AP) configurations, and (b) dependence of the TMR, as a function of Au layer thickness. 


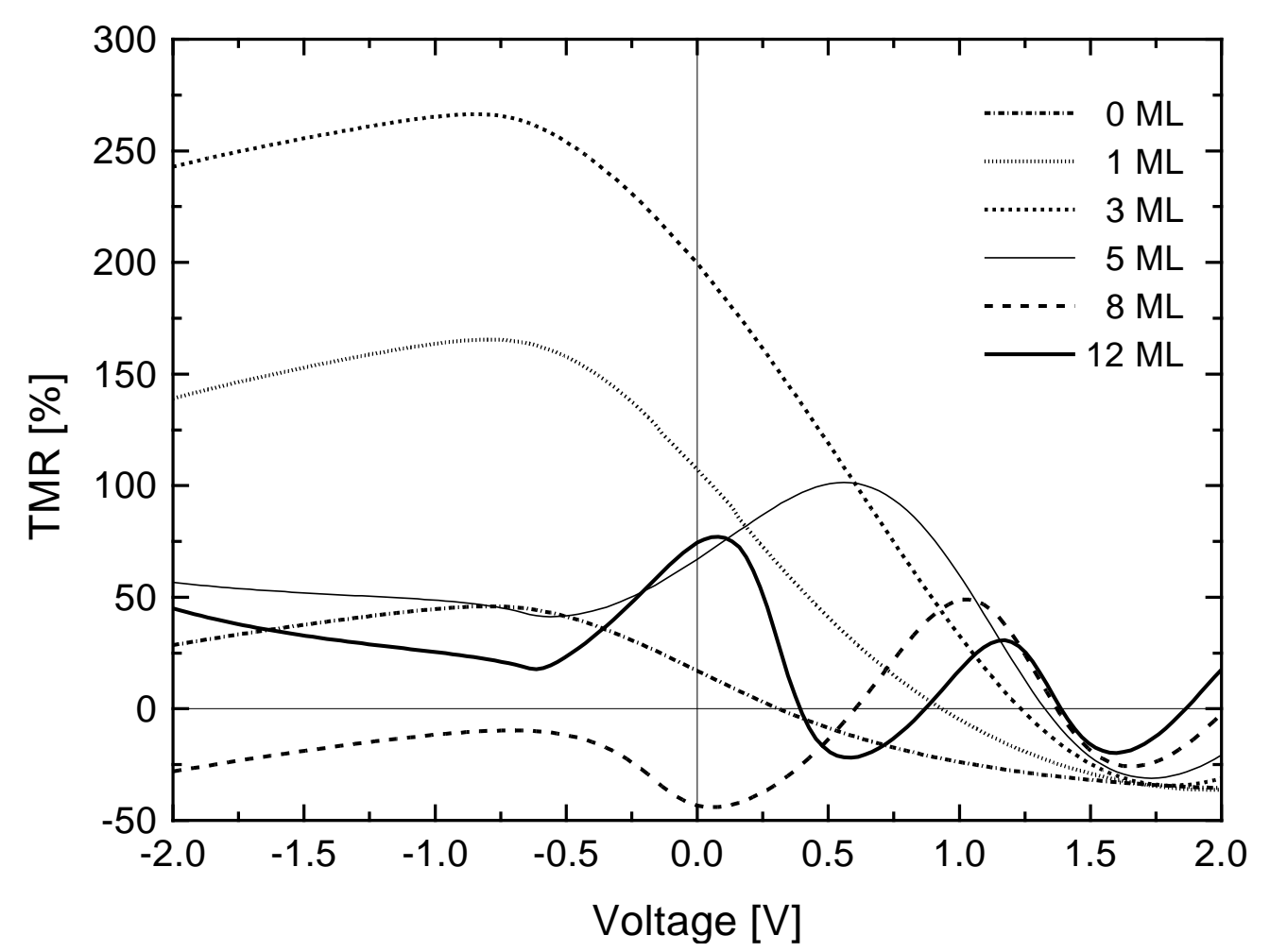

FIG. 3. Dependence of the TMR on the applied voltage for different NM multilayers.

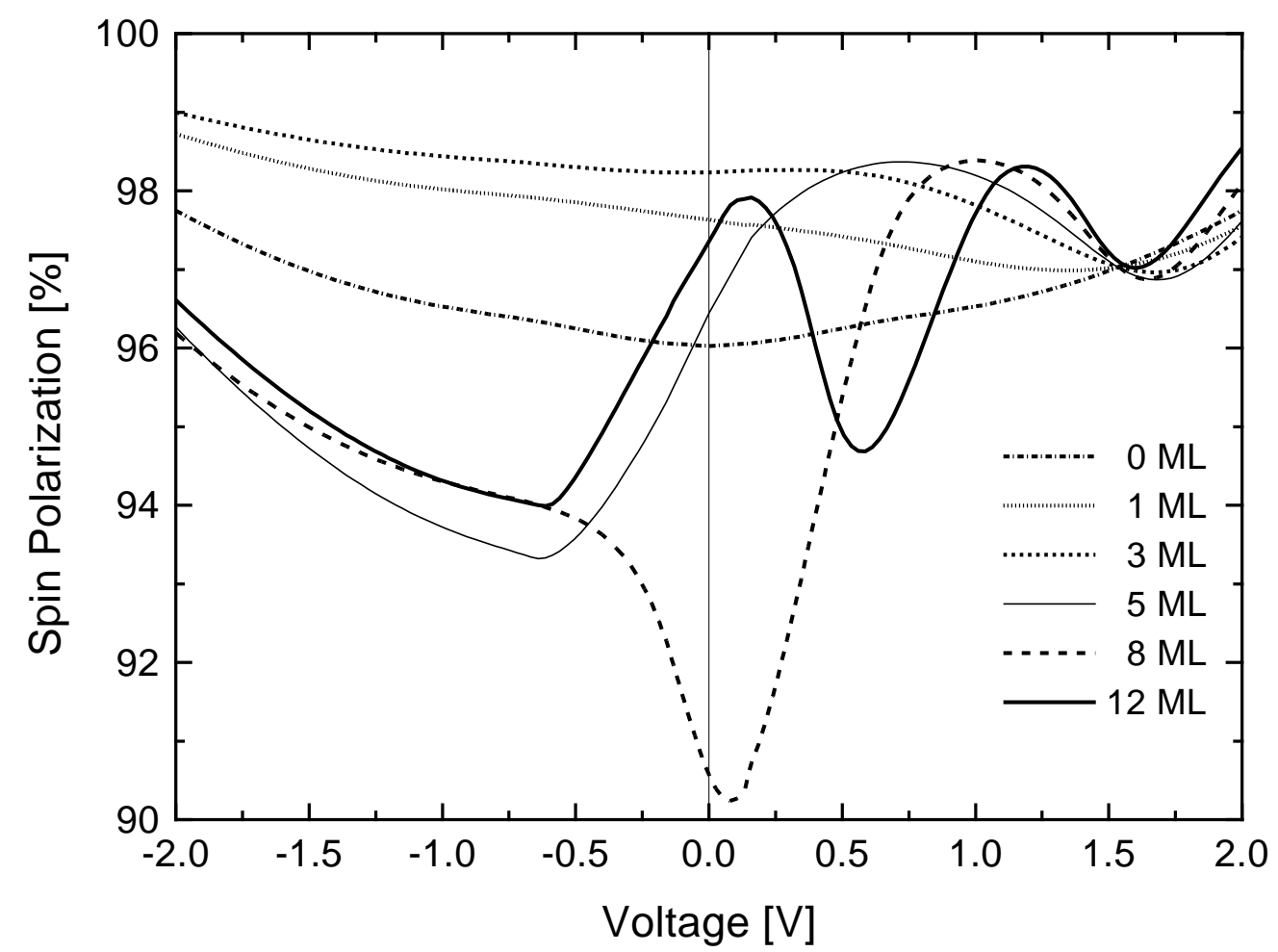

FIG. 4. Dependence of the spin polarization on the applied voltage for different NM multilayers. 